\title{
IMPORTÂNCIA DOS ASPECTOS NUTRICIONAIS COMO FATOR ASSOCIADO À SÍNDROME DE HIPERATIVIDADE COM DÉFICIT DE ATENÇÃO
}

\author{
ANA GUAARIOLA*, FLÁVIO D. FUCHS** ALEX R. TERRA***, CLAUDIA CUNHA****,

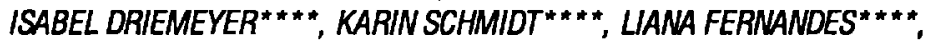 \\ JAOUELINE LEAL****, NEWRA T. ROTTA*****
}

\begin{abstract}
RESUMO - O distúrbio de hiperatividade com déficit de atenção constitui síndrome comum na infância, pelo que é reconhecido como problema médico-social importante, sendo objeto de extensa investigaçăo quanto aos seus determinantes, abordagens diagnosticas e formas de prevenção e tratamento. Dentro dos fatores que podem alterar a função cerebral, encontra-se a desnutrição. Os autores discutem a associação da sindrome com o estado nutricional de uma população de escolares.
\end{abstract}

PALAVRAS CHAVE: distúrbio de hiperatividade com déficit de atençăo, desnutriçăo.

Nutritional aspects importance in attention deficit hyperactivity disorder

ABSTRACT - The attention deficit hyperactivity disorder is a common childhood syndrome that is recognized as an important medical-social problem, being the object of an exhaustive investigation related to its etiology, diagnostic approach and prevention, and treatment forms. Among the factors that can alter the cerebral function, is the malnutrition. The authors discuss the association of the syndrome with the nutritional condition of students from elementary schools.

KEY WORDS: attention deficit hyperactivity disorder, malnutrition.

A síndrome de hiperatividade com déficit de atenção (SHDA) é síndrome comum na infância, pelo que é reconhecida como problema médico-social importante, sendo objeto de extensa investigaçāo quanto a seus determinantes, abordagens diagnósticas e formas de prevenção e tratamento. A síndrome se caracteriza por alterações dos sistemas motores, perceptivos, cognitivos e do comportamento, comprometendo a aprendizagem de crianças com potencial intelectual adequado 9,13,18,19. Esta entidade tem apresentado denominaçōes diferentes no decorrer do tempo. Strauss e Lehtinen, em 1947, chamavam este quadro de "lesāo mínima". No simpósio realizado em Oxford , em 1962, foi oficializada a expressão "đisfunção cerebral mínima"'1. Em 1980, passou-se a empregar a expressão "distúrbio de atenção" com ou sem hiperatividade (DSM-III, 1980) ${ }^{1}$ mudando para "distúrbio de hiperatividade com déficit de atenção"(DSM-III-R, 1987)2 , denominação que se mantém no DSM-IV (1994)".

*Livre Docente em Neurologia Infantil, Fundação Faculdade Federal de Ciências Médicas de Porto Alegre (FFCMPA); **Livre Docente em Cardiologia, FFCMPA; ***Doutorando da FFCMPA; ****Medica Neurologista; *****Médica Pediatrica; ******Livre Docente em Neurologia, Faculdade de Medicina da Universidade Federal do Rio Grande do Sul (UFRGS). Aceite: 22-maio-1997.

Dra. Ana Guardida - Departamento de Neurologia - Fundação Faculdade Federal de Ciências Médicas do Rio Grande do Sul - Rua Sarmento Leite 245 - 90050-140 Porto Alegre RS - Brasil. 
Em relação à etiologia, o SHDA pode advir de fatores endógenos e exógenos (Fig 1), responsáveis pelo comprometimento cerebral, manifestando-se por alteraçð̋es funcionais ${ }^{11,13,18,19}$. Salienta-se dentro dos fatores exógenos a desnutrição, um problema de saúde pública em países subdesenvolvidos. Este fator age no cérebro da criança desde a etapa pré-natal, produzindo perda de células cerebrais que, dependendo da intensidade e da idade, acarretará danos irreversíveis. A desnutrição precoce afeta permianentemente o crescimento do cérebro enquanto que a mais tardia pode determinar crescimento impróprio passível de recuperação. A desnutrição pode gerar atraso do desenvolvimento psicomotor, intelectual e consequentemente dificuldades de aprendizado ${ }^{7,14,15,17,22}$. Na espécie humana, o período de aceleração do crescimento cerebral estende-se desde a trigésima semana de gestaçāo até, pelo menos, o final do segundo ano de vida. Neste perfodo existe maior probabilidade de dano cerebral permanente ${ }^{7}$. Winick ${ }^{22}$ mostrou que: a má nutrição pré-natal determina um déficit de $15 \%$ das células cerebrais; a má nutrição no $1^{\circ}$ ano de vida determina também um déficit de $15 \%$ e que as duas situaçöes associadas elevam esta percentagem para $60 \%$. Cravioto estudando crianças desnutridas durante o primeiro ano de vida, constatou que aquelas que apresentavam peso normal ao nascer tinham déficit de $15 \%$ das células cerebrais e as que apresentavam baixo peso ao nascer tinham déficit de $60 \%$ de células nervosas ${ }^{6}$.

O objetivo desta pesquisa foi avaliar a relação entre o estado nutricional e a síndrome de SHDA.

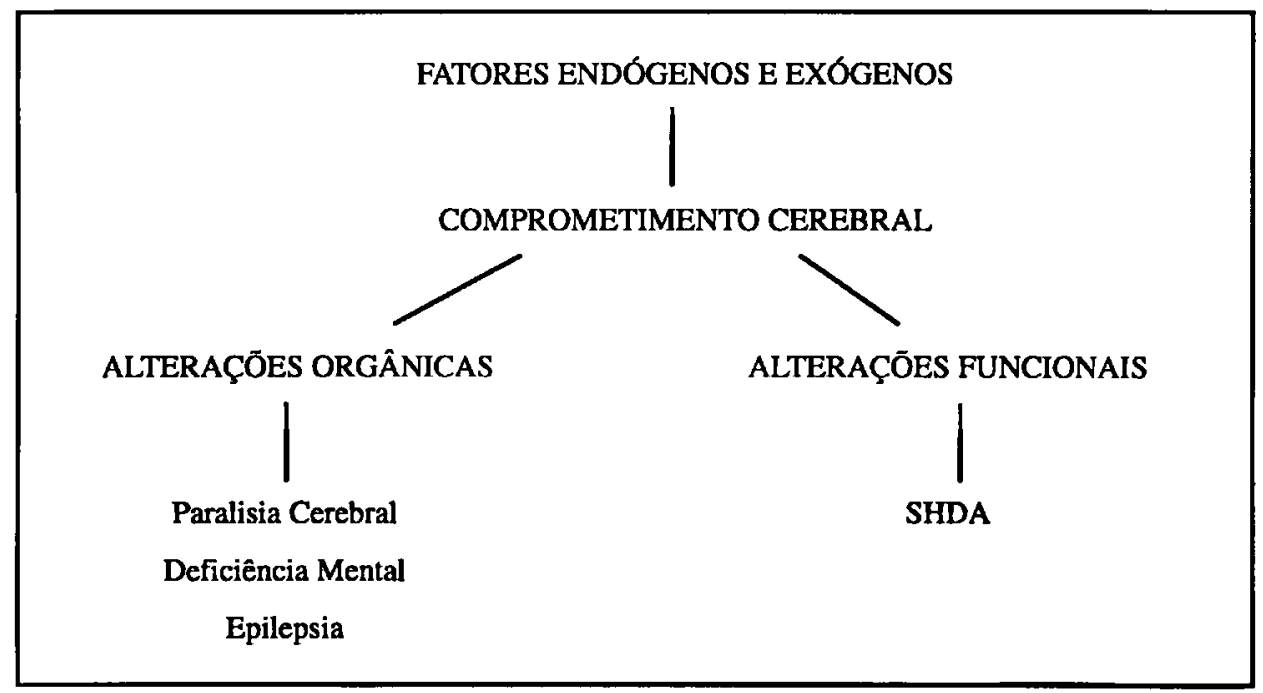

Fig l. Etiologia do SHDA.

\section{MÉTODOS}

Foi realizado estudo observacional, analítico e de delineamento transversal, sendo utilizada amostragem aleatória e proporcional de todos os escolares $(n=35521)$ de $1^{2}$. série, distribuídos em escolas estaduais ( $n=22966$ ), particulares $(n=8311)$ e municipais $(n=4244)$.

O tamanho da amostra foi calculado estimando uma precisāo de medida $\pm 3,0 \%$,correspondendo ao intervalo de confiança de $95 \%$, para uma prevalência prevista de $10 \%$. Assim, a amostra ficou constituída de 310 crianças da rede estadual, 58 da rede municipal e 116 crianças das escolas particulares, totalizando 484 crianças.

Aferiu-se sexo, idade, cor, estado nutricional, escola de origem.

O exame neurológico foi feito em todas as crianças, avaliando-se psiquismo, linguagem, fascies, atitude, equilíbrio, tono, motricidade voluntária, motricidade reflexa, motricidade passiva, sensibilidáde e nervos craneanos. 
Definiu-se como normal o exame que tinha, como máximo, alteração do tono e da linguagem. As crianças que năo preencheram este critério foram exclufdas.

O exame neurológico evolutivo (ENE), pelo qual se avaliou linguagem, lateralidade, equilíbrio estático, equilíbrio dinâmico, coordenaçăo apendicular, persistência motora, sensibilidade e gnosias, e coordenaçăo troncomembros, foi usado para avaliar a maturidade neurologica. Aplicaram-se também os subtestes números, completar figuras e código da escala de Wisc.

O comportamento foi avaliado pelos critérios do DSM-IV ${ }^{3}$, confeccionando com este critério um questionário respondido pela professora.

Foram empregados três critérios diagnósticos: o primeiro, a partir daqui denominado SHDA 1, baseou-se na proposta do DSM-IV; o segundo SHDA 2, baseou-se nos critérios neuropsicológicos apresentados no quadro 1, contemplando o ENE discrepante, alteraçóes psicométricas, do comportamento e do aprendizado; o SHDA 3 seguiu os mesmos criterios do SHDA 2, exceto quanto ao item 13, em que foi considerada somente a avaliação da persistência motora.

O estado nutricional foi avaliado através dos escores que representam o número de desvios-padrảo abaixo ou acima da mediana da população. Dos trés índices: altura para a idade, peso para a idade e peso para a altura; os dois primeiros fornecem dados sobre desnutrị̧a crónica e o último sobre desnutriçăo aguda As crianças foram consideradas desnutridas quando apresentavam indice menor ou igual do que dois desvios-padräo negativos (-2DP) ${ }^{21}$.

Foi obtido consentimento das Secretarias de Educagão do Estado e do Município e da Diregäo das escolas particulares.

As crianças foram avaliadas individualmente na própia escola. $O$ tempo usado para avaliaçăo foi em torno de 60 minutos. A equipe estava constituída de 10 médicos.

Aplicou-se o teste qui-quadrado para avaliar a significância das associaçð̃es entre variáveis categóricas. Usou-se a ANOVA para variáveis contínuas. Considerou-se o $\mathrm{P}$ alfa $5 \%$ significante (bicaudal).

Testou-se a associaçăo bruta de sexo, idade, cor, tipo de escola e estado nutricional com SHDA 1, SHDA 2 e SHDA 3.

Quadro 1. Critérios diagnósticos do SHDA no periodo escolar, segundo aspectos neunopsicológicos.

1. Hiperatividade

2. Coordenaçăo motora pobre

3. Dificuldade em concentrar-se

4. Dificuldade de atençăo

5. Sentado, mexendo em pequenos objetos

6. Interfere nas atividades de outras crianças

7. Temperamento explosivo

8. Teme năo ser aceito pelos colegas

9. Perturba outras crianças

10. Dificuldade de aprendizado

11. Exame neurológico sem assimetrias

12. Alteraçăo do tono: hipotonia ou paratonia

13. Exame neurológico evolutivo discrepante, desenvolvimento abaixo do esperado em equilíbrio estático, coordenaçăo apendicular, sensibilidade e gnosias, persistência motora

14. Alteraçōes nos subtestes números, completar figuras e código da escala de WISC 


\section{RESULTADOS}

e estatura.

A Tabela 1 mostra a distribuição das crianças por escola e por sexo e a Tabela 2 a idade, peso

Tabela 1. Distribuição das crianças por escola e por sexo.

\begin{tabular}{lcccccc}
\hline Escola & \multicolumn{2}{c}{ Meninos } & \multicolumn{2}{c}{ Meninas } & \multicolumn{2}{c}{ Total } \\
& N & $\%$ & N & $\%$ & N & $\%$ \\
\hline Estadual & 145 & 46,8 & 165 & 53,2 & 310 & 64,7 \\
Municipal & 25 & 43,1 & 33 & 56,9 & 58 & 11,9 \\
Particular & 69 & 59,5 & 47 & 40,5 & 116 & 23,4 \\
Total & 239 & 49,4 & 245 & 50,6 & 484 & 100 \\
\hline
\end{tabular}

$\times 2=6,49 ; P=0,039 *$

* $P$ com significaçāo estatística

Tabela 2. Idade, peso e estatura por sexo: média $(D P)$.

\begin{tabular}{lccccc}
\hline Características & Amostra & Meninos & Meninas & $F^{*}$ & $P$ \\
\hline Idade (meses) & 89,5 & 90,3 & 88,3 & 4,37 & $0,038^{*}$ \\
& $(11,0)$ & $(11,9)$ & $(9,9)$ & & \\
Peso (kg) & 26,1 & 26,1 & 26,0 & 0,04 & 0,839 \\
& $(5,6)$ & $(5,8)$ & $(5,5)$ & & \\
Estatura (cm) & 126,8 & 127,0 & 126,6 & 0,23 & 0,632 \\
& $(7,3)$ & $(7,8)$ & $(7,9)$ & & \\
\hline
\end{tabular}

" $P$ com significą̧ăo estatística

A prevalência dos indicadores de desnutrição classificada por escola e por sexo está apresentada nas Tabelas 3 e 4.

Tabela 3. Prevaléncia de défict de altura para a idade (A/), peso para idade (P/I) e peso para altura (P/A) por categoria de escola.

\begin{tabular}{ccccccccc}
\hline Indices & \multicolumn{2}{c}{ Estadual } & \multicolumn{2}{c}{ Municipal } & \multicolumn{2}{c}{ Particular } & $\times 2$ & P \\
& $\mathrm{N}$ & $\%$ & $\mathrm{~N}$ & $\%$ & $\mathrm{~N}$ & $\%$ & & \\
\hline A/I & 11 & 3,6 & 1 & 1,7 & zero & zero & 4,57 & 0,102 \\
P/I & 7 & 2,3 & 2 & 3,4 & 1 & 0,9 & 1,44 & 0,488 \\
P/A & 10 & 3,2 & 6 & 10,3 & 1 & 0,9 & 10,44 & $0,005^{*}$ \\
\hline
\end{tabular}


Tabela 4. Prevalência de déficit de altura para idade (AI), peso para idade (P/I) e peso para a altura (P/A) por sexo.

\begin{tabular}{|c|c|c|c|c|c|c|}
\hline \multirow[t]{2}{*}{ Indices } & \multicolumn{2}{|c|}{ Masculino } & \multicolumn{2}{|c|}{ Feminino } & \multirow[t]{2}{*}{$x 2$} & \multirow[t]{2}{*}{$\mathbf{P}$} \\
\hline & $\mathbf{N}$ & $\%$ & $\mathrm{~N}$ & $\%$ & & \\
\hline $\mathrm{A} / \mathrm{I}$ & 8 & 3,4 & 4 & 1.6 & 0,86 & 0,353 \\
\hline $\mathbf{P} / \mathbf{I}$ & 5 & 2,1 & 5 & 2,0 & 0,00 & 1.000 \\
\hline P/A & 10 & 4,2 & 7 & 2,9 & 0,30 & 0,579 \\
\hline
\end{tabular}

Como se pode observar, as crianças matriculadas nas escolas municipais apresentaram mais frequentemente déficit de peso para a altura. Nāo houve diferença nos demais índices entre as escolas e os sexos.

A Tabela 5 mostra a prevalência do SHDA e os respectivos intervalos de confiança segundo os diferentes critérios diagnósticos. Mais crianças tem SHDA segundo as propostas pelo DSM-IV (SHDA 1) do que pelos critérios neuropsicológicos (SHDA 2 e SHDA 3).

Tabela 5. Prevalência de SHDA segundo critérios diagnósticos $(N=484)$.

\begin{tabular}{llll}
\hline Criterio & N & $\%$ & IC \\
\hline SHDA 1 & 87 & 18,0 & $14,6-21,4$ \\
SHDA 2 & 17 & 3,5 & $1,5-5,5$ \\
SHDA 3 & 19 & 3,9 & $1,0-5,9$ \\
\hline
\end{tabular}

A Tabela 6 mostra a prevalência de SHDA 1, SHDA 2, SHDA 3 por escola, observando-se que na escola municipal havia mais crianças com SHDA 3. A distribuição de SHDA por sexo é encontrada na Tabela 7.

Tabela 6. Prevalência de SHDA segundo os diferentes critérios diagnósticos por escolas.

\begin{tabular}{lcccccccccc}
\hline SHDA & \multicolumn{2}{c}{ Total } & \multicolumn{2}{c}{ Estadual } & \multicolumn{2}{c}{ Municipal } & \multicolumn{2}{c}{ Particular } & x2 & P \\
& $\mathrm{N}$ & $\%$ & $\mathrm{~N}$ & $\%$ & $\mathrm{~N}$ & $\%$ & $\mathrm{~N}$ & $\%$ & & \\
\hline SHDA 1 & 87 & 18,0 & 56 & 18,1 & 15 & 25,9 & 16 & 13,8 & 4,50 & 0,212 \\
SHDA 2 & 17 & 3,5 & 12 & 3,9 & 4 & 6,9 & 1 & 0,9 & 4,48 & 0,106 \\
SHDA 3 & 19 & 3,9 & 13 & 4,2 & 5 & 8,6 & 1 & 0,9 & 6,33 & $0,042^{*}$ \\
\hline
\end{tabular}

*P com significação estatística

Tabela 7. Prevalência de SHDA segundo os diferentes critérios diagnósticos por sexo.

\begin{tabular}{ccccccccc}
\hline SHDA & \multicolumn{2}{c}{ Total } & \multicolumn{2}{c}{ Meninos (239) } & \multicolumn{2}{c}{ Meninas (245) } & x2 & P \\
& N & $\%$ & N & $\%$ & N & $\%$ & N & $\%$ \\
\hline SHDA 1 & 87 & 18,0 & 60 & 25,1 & 27 & 11,0 & 15,33 & $<0,001^{*}$ \\
SHDA 2 & 17 & 3,5 & 8 & 3,3 & 9 & 3,7 & 0,00 & 1,000 \\
SHDA 3 & 19 & 3,9 & 10 & 4,2 & 9 & 3,7 & 0,00 & 0,956 \\
\hline
\end{tabular}


As tabelas 8, 9 e 10 correlacionam os índices de nutrição com o diagnóstico de SHDA. Por qualquer critério, as crianças com o distúrbio tinham menores índices de altura para a idade do que as sem SHDA.

Tabela 8. Média (DP) do índice altura para a idade (NI), em desvios-padrōes da normalidade, por SHDA.

\begin{tabular}{ccccc}
\hline Critérios & Condição (n) & AVI & F & P \\
\hline SHDA 1 & Sim (87) & $0,23(1,28)$ & 9,21 & $0,002^{*}$ \\
& Não (397) & $0,78(1,58)$ & & \\
SHDA 2 & Sim (17) & $-0,13(0,93)$ & 4,94 & $0,027^{*}$ \\
& Não (467) & $0,71(1,56)$ & & \\
SHDA 3 & Sim (19) & $-0,17(0,89)$ & 6,11 & $0,013^{*}$ \\
& Não (465) & $0,71(1,56)$ & 6,11 & $0,013^{*}$ \\
\hline
\end{tabular}

*P com significação estatística

Tabela 9. Média (DP) do índice peso para a idade (P/I), em desvios-padrōes da normalidade, por SHDA.

\begin{tabular}{ccccc}
\hline Critério & Condição (n) & P/1 & F & P \\
\hline SHDA 1 & Sim (87) & $0,32(1,9)$ & 2,92 & 0,088 \\
& Não (397) & $0,65(1,6)$ & & \\
SHDA 2 & Sim (17) & $0,29(1,50)$ & 2,03 & 0,154 \\
& Não (467) & $0,61(1,60)$ & & \\
SHDA 3 & Sim (19) & $-0,03(1,40)$ & 2,79 & 0,095 \\
& Não (465) & $0,61(1,70)$ & & \\
\hline
\end{tabular}

Tabela 10. Média (DP) do indice peso para a altura (P/A), em desvios-padrōes da normalidade, por SHDA.

\begin{tabular}{ccccc}
\hline Critérios & Condição (n) & P/A & F & P \\
\hline SHDA 1 & Sim (87) & $0,40(2,3)$ & 0,19 & 0,657 \\
& Não (397) & $0,50(2,4)$ & & \\
SHDA 2 & Sim (17) & $0,11(1,50)$ & 0,40 & 0,525 \\
& Não (467) & $0,48(2,40)$ & & \\
SHDA 3 & Sim (19) & $0,10(1,50)$ & 0,53 & 0,464 \\
& Não (465) & $0,48(2,80)$ & & \\
\hline
\end{tabular}




\section{DISCUSSÃO}

A acentuada diferença nas taxas de prevalência de SHDA em crianças de 1*. série de Porto Alegre, na dependência do emprego dos critérios do DSM-IV, sugere fortemente que o emprego deste critério superestima a prevalência da doença. Esta interpretação concorda com a de August e Garfinkel ${ }^{4}$ que recomendam a utilização do DSM-III-R somente para "screening" de SHDA. Baumgaertel e col. ${ }^{5}$ mostram que os critérios do DSM-IV também servem como "screening", mas não são decisivos para o diagnóstico de SHDA.

Estudar o estado nutricional de uma população infantil como fator associado ao SHDA é da maior importância. $O$ estado nutricional de uma criança depende de muitos fatores: peso de nascimento, episódios de infeç̧ão, internaçōes hospitalares, espaçamento intergestacional, disponibilidade adequada de alimentos em qualidade e quantidade, idade da mãe, escolaridade e ocupaçāo dos pais e condiçōes de moradia ${ }^{10,12,16}$.

Cravioto e De Licardie $e^{6}$ avaliaram a influência do ambiente na ocorrência de desnutrição em um grupo de 22 crianças que desenvolveram desnutrição proteico-calórica severa comparado com um grupo sem desnutrição. Observaram que a presença de desnutrição severa entre as crianças das familias de maior risco está associada a microambiente francamente inadequado, caracterizado por baixo nivel de estimulação no lar e mãe mais passiva que não percebe as necessidades de seu filho.

As crianças que sobrevivem à desnutrição adquirem padrão de alimentação que cada vez mais lhes permite melhores probabilidades de sobrevida e de crescimento, com um estado nutricional que, mesmo não sendo adequado, não será tão prejudicial como seria nas primeiras etapas de sua vida?

Cerca de 20\% dos desnutridos brasileiros, quando chegam à idade adulta, entre 20 e 25 anos, são classificados como "nanicos"(Ministério de Saúde, Instituto Nacional de Alimentação e Nutrição, 1990). Ao entrar no mercado de trabalho, os adultos desnutridos terão limitaçōes no seu desempenho, repercutindo na produtividade e consequentemente em seus ganhos. $O$ destino desse trabalhador será residir na periferia das cidades e ter filhos desnutridos ${ }^{12.20}$.

Neste estudo, as crianças que tinham índices mais baixos de nutriçāo mostraram associação com a síndrome de SHDA por qualquer critério.

O fato de se observar maior índice de prevalência de SHDA nas escolas municipais se deve à presença de crianças com pior índice de nutrição.

\section{CONCLUSŌES}

O estudo permitiu descrever, com acuidade, as taxas de prevalência do distúrbio de hiperatividade com déficit de atenção em escolares da $1^{2}$. série de Porto Alegre, permitindo concluir que:

1. As taxas de prevalência do distúrbio diagnosticado pelos critérios neuropsicológicos são acentuadamente menores do que as detectadas pelos critérios comportamentais.

2. Na composição dos critérios neuropsicológicos diagnósticos da síndrome, a utilização da persistência motora alterada detecta praticamente todos os casos diagnosticados com o emprego do exame neurológico evolutivo discrepante. do DSM-IV.

3. A prevalência de SHDA é maior entre os meninos somente quando se empregam os critérios

4. $O$ índice altura para a idade é menor entre as crianças com SHDA diagnosticadas por qualquer abordagem. $O$ índice peso para a idade tende a ser menor entre as crianças com a síndrome diagnosticada pelos critérios comportamentais e neuropsicológicos. Isto sugere que a desnutrição crônica é um fator de risco para o SHDA. 


\section{BIBLIOGRAFIA}

1. American Psychiatric Association. Diagnostic and statistical manual of mental disorders (DSM-III). Washington DC: American Psychiatric Association, 1980.

2. American Psychiatric Association. Diagnostic and statistical manual of mental disorders (DSM-III-R). Washington DC: American Psychiatric Association, 1987.

3. American Psychiatric Association. Diagnostic and statistical manual of mental disorders (DSM-IV). Washington DC: American Psychiatric Association, 1994.

4. August GJ, Garfinkel BD. The nosology of attention-deficit hyperactivity disorder. J Am Acad Children Adolesc Psychiatry 1993:32:155-165.

5. Baurmgaertel A, Wolraich ML, Dietrich M. Comparison of diagnostic criteria for attention deficit disorders in a German elementary school sample, J Am Acad Children Adolesc Psychiatry 1995;34:629-638.

6. Cravioto J, De Licardie ER. Enviromental correlates of severe clinical malnutrition and language developmental in survivors from kwashiorkor or marasmus. Bol Ofic Sanit Panamer (English Edition) 1973;7:50-55.

7. Cravioto J, Arrieta Milan R. Má nutrição e sistema nervoso central. In Lefrève AB, Diament A, Cypel S. Neurologia infantil. Ed2. Sāo Paulo: Atheneu, 1989:1207-1231.

8. Drillien CM. The growth and development of the prematurely born infant. London: E \& S. Livingstone, 1964.

9. Fischer W, Burd L, Kuna D, Berg D. Attention deficit disorders and the hyperactivity in multiple disabled children. Rehabilitation Literature 1985:46:250-254.

10. Giugliani ERJ, Rotta AT, Ribeiro AM, Mello C, Moreira C, Dias CCC, Prytalux TM. Percepção materna sobre adequação do peso e da altura de crianças menores de 5 anos em uma vila periférica de Porto Alegre. Revista HCPA 1990;10:70-73.

11. Guardiola A. Uso da imipramina no estudo da hiperatividade com déficit de atençāo. Tese. Fundação Faculdade Federal de Ciências Médicas de Porto Alegre. Porto Alegre: 1990.

12. Issler RMS. Niveis de pobreza e sua repercusăo no estado de saúde de populaçóes urbanas de baixa renda: um estudo em Porto Alegre RS. Tese, Faculdade de Medicina da Universidade Federal do Rio Grande do Sul. Porto Alegre, 1993.

13. Lefrève AB. Disfunçāo cerebral minima: estudo multidiciplinar. São Paulo: Sarvier, 1975.

14. Lefrève AB, Diament A, Cypel S. Neurologia infantil. Ed2. São Paulo: Atheneu, 1989.

15. Marcondes E, Lefrève AB, Machado DVM. Desenvolvimento neuropsicomotor da criança desnutrida. Rev Bras Psiquiatria, 1969;3:173-219.

16. Monckberg F. Desnutrición infantil : fisiopatologia, clinica, tratamiento y prevención: nuestra experiencia y contribuición. Santiago Impresora Creces, 1988.

17. Pollit E. Desnutricion y rendimiento escolar. In Cuzminsky M, Moreno EM, Ojeda ENS. Crescimiento y desarrolo: hechos y tendencias. Washington: OPAS, 1988;324-348.

18. Rebollo MA. Etiologia de la disfunción cerebral minima. Neuropediatria Latinoamer, 1972;1:17.23.

19. Rotta NT. Avaliação neurológica evolutiva, eletroencéfalográfica e psicológica em crianças com rendimento escolar deficiente. Tese, Fundaçāo Católica de Medicina Porto Alegre, 1975.

20. Spurr GB. Tamaño corporal, capacidad de realizar trabajos físicos y productividad en el trabajo intenso: es mejor más grande? In Retraso del crescimiento lineal en los paises en vias de desartollo. Brasil: Nestlé Nutrition, 1987:25-29.

21. WHO Working Group. Use and interpretation of anthropometric indicators of nutritional status. Bull World Health Organiz; 1986;64:924-941.

22. Winick M. Cellular growth during early malnutrition. Pediatrics, 1971;47:969-972. 